\title{
Impact of COVID-19 pandemic on doctors' clinical practice
}

\author{
Naveen S Kulkarni', Neelakanth S Patil'2, Vishalakshi ${ }^{3}$, Asha Bullappa ${ }^{4}$, \\ Hithashree Shriyan ${ }^{5}$, Subhashree Mishra ${ }^{6}$ \\ ${ }^{1,2}$ Associate Professor, ${ }^{5,6}$ Post Graduate Resident, Department of General Medicine, ${ }^{3}$ Assistant Professor, \\ Department of Ophthalmology, Shri Dharmasthala Manjunatheshwara College of Medical Sciences and Hospital, Shri \\ Dharmasthala Manjunatheshwara University, Dharwad, ${ }^{4}$ Associate Professor, Department of Community Medicine, SS \\ Institute of Medical Sciences and Research Centre, Davangere, Karnataka, India
}

Background: The pandemic of COVID-19 has affected doctors, nurses, patients, and people working in hospitals. Aims and Objectives: This study aims to study the trends in doctors' clinical practice during COVID-19 pandemic. Materials and Methods: The study conducted at SDMCMS\&H, Dharwad, includes questionnaire on the impact of COVID-19 pandemic on doctors'clinical practice. The experience of doctors between May 2020 and June 2021 working in SDM University is considered in this study. Results: The number of non-COVID patients in OPD and IPD has reduced. The time spent in clinical examination is reduced, reliability on investigations has increased. The use of telemedicine is increasing. Conclusion: The impact of COVID-19 on clinical practice and doctors is multifactorial.The doctors and other health care workers can modify the COVID-19 impact on clinical practice by continuing to deliver the services as during pre-pandemic.

Key words: Clinical; COVID; Pandemic; Practice; Telemedicine
Access this article online

Website:

http://nepjol.info/index.php/AJMS

DOI: 10.3126/ajms.v13i3.40909

E-ISSN: 2091-0576

P-ISSN: 2467-9100

Copyright (c) 2022 Asian Journal of Medical Sciences

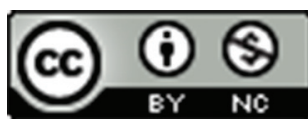

This work is licensed under a Creative Commons Attribution-NonCommercial 4.0 International License.

\section{INTRODUCTION}

A cluster of "atypical viral pneumonia" cases was diagnosed in Wuhan City, China, in December 2019. By January 9, 2020, Chinese authorities found that the cause of the outbreak was a novel coronavirus - later named COVID-19 which was later called severe acute respiratory syndrome coronavirus 2 (SARS-CoV-2). ${ }^{1,2}$

The World Health Organization declared an international public health emergency. The countries across the world commenced national lockdown, travel ban, schools, colleges, and universities were indefinitely closed and recommended that health care workers observe physical distancing, wear personal protective equipment, and use telephone triage and appointments to reduce face-to-face contact. $^{1,2}$

India reported the first case of this coronavirus disease (COVID-19) on January 30, 2020, in Kerala, where a student with a history of travel to Wuhan presented with respiratory symptoms. ${ }^{2}$

The proposed modes of human to human transmission in SARS-CoV-2 were through droplets, fomites, and contacts. The manifestations are fever, cough, sore throat, fatigue, dyspnea, diarrhea, and vomiting, and in select group, it may lead to acute respiratory distress syndrome, septic shock, multiorgan failure, and death. ${ }^{2}$ 
The pandemic has affected the hospital functioning and patient care. ${ }^{1-4}$

Doctors and health care workers are the frontline workers in the battle against ongoing pandemic. We conducted this survey to study the impact of COVID-19 pandemic on doctors' clinical practice.

\section{Aims and objectives}

This study aims to study the trends in the clinical practice of doctors due to COVID-19 pandemic.

\section{MATERIALS AND METHODS}

This study is conducted at Shri Dharmasthala Manjunatheshwara College of Medical Sciences and Hospital, Shri Dharmasthala Manjunatheshwara University, Dharwad, Karnataka, India.

It is a cross-sectional qualitative study.

\section{Inclusion criteria}

The participants of this study are consultant doctors and resident doctors of all the medical and dental clinical branches working in Shri Dharmasthala Manjunatheshwara University, Dharwad.

\section{Exclusion criteria}

The doctors of preclinical and paraclinical departments working in Shri Dharmasthala Manjunatheshwara University, Dharwad.

One hundred and eighteen doctors are participants of this study. The study period is from May 2020 to June 2021. The experiences in clinical practice during this period are considered.

\section{Materials}

The study includes the questionnaire on the impact of COVID-19 pandemic on doctors' clinical practice.

\section{Method of data collection}

The questionnaire "Clinical practice patterns during COVID-19 pandemic - a study" was given to all participants in the form of hard copy and the responses were noted down. The questionnaire included 25 questions with multiple options. The consent form for the same was given with the questionnaire.

\section{Ethics}

Ethical committee clearance was obtainedfrom the Institutional Ethics Committee of SDMCMS\&H, SDM University, Dharwad[Ref: SDMIEC/2021/01 Date: 15/07/21].

\section{Statistical method}

Data were entered into the Excel spreadsheet. Descriptive statistics of the explanatory and outcome variables were calculated by mean, standard deviation for quantitative variables, frequency, and proportions for qualitative variables.

\section{RESULTS}

Our study noted the responses of 118 doctors. Sixty-four $(54.2 \%)$ were female respondents and $54(45.8 \%)$ were male.

The doctors included were from various clinical specialties from medical college, dental college, and physiotherapy college of SDMCMS\&H, SDM University, Dharwad. Fiftytwo doctors (44.1\%) were from surgical branches. Sixty-six doctors $(55.9 \%)$ were from medical branches.

The doctors of all cadres were included in the study professors, associate professors, assistant professors, senior residents, junior residents, and interns. The participants were 68 junior residents $(57.6 \%), 6(5.1 \%)$ senior residents, $20(16.9 \%)$ assistant professors, $5(4.2 \%)$ associate professors, and 11(9.3\%) were professors.

The average number of OPD cases seen by doctors prepandemic and during pandemic is shown in the following Table 1.

The average number of cases seen by doctors in IPD prepandemic and during pandemic is shown in the following Table 2.

The cases seen by doctors, in their concerned departments, during the pandemic are shown in the Table 3.

\begin{tabular}{lcc}
$\begin{array}{l}\text { Table 1: The average number of OPD cases } \\
\text { seen by doctors' pre pandemic and during } \\
\text { pandemic }\end{array}$ \\
\hline OPD cases per week & Pre-pandemic & During pandemic \\
\hline$<20$ & $6(5.1 \%)$ & $48(40.7 \%)$ \\
$20-50$ & $23(19.5 \%)$ & $46(39 \%)$ \\
$50-100$ & $33(28 \%)$ & $22(18.6 \%)$ \\
More than 100 & $53(44.9 \%)$ & $1(0.8 \%)$ \\
\hline
\end{tabular}

\begin{tabular}{lcc}
$\begin{array}{l}\text { Table 2: The average number of cases seen } \\
\text { by doctors in IPD pre pandemic and during } \\
\text { pandemic }\end{array}$ \\
\hline Cases - IPD per week & Pre-pandemic & During pandemic \\
\hline$<20$ & $21(17.8 \%)$ & $68(57.6 \%)$ \\
$20-50$ & $52(44.1 \%)$ & $32(27.1 \%)$ \\
$50-100$ & $15(12.7 \%)$ & $12(10.2 \%)$ \\
More than 100 & $25(21.2 \%)$ & $6(5.1 \%)$ \\
\hline
\end{tabular}


The time given by doctors to the patients during this pandemic in OPD and IPD is shown in the following Table 4.

The time given by doctor to the patient during the pandemic (OPD and IPD): Table 5.

The changes noted in procedures or surgeries by doctors during this pandemic, in comparison to pre-pandemic, are shown in the following Table 6.

Telemedicine advice given doctors during the pandemic: Table 7.

The doctors' opinion on telemedicine is shown in Table 8.

The type of personal protection measures used by doctors during the pandemic isshown in the Table 9) (OPD and IPD).

\begin{tabular}{lc} 
Table 3: The cases seen by doctors, in their \\
concerned departments, during the pandemic \\
\hline Cases seen in pandemic & $\mathbf{n}$ \\
\hline SARI/COVID related & $51(43.2 \%)$ \\
emergency & $23(19.5 \%)$ \\
Referrals & $14(11.9 \%)$ \\
Cross-consultations & $12(10.2 \%)$ \\
Same as pre-pandemic & $18(15.3 \%)$ \\
\hline
\end{tabular}

\begin{tabular}{lcc}
\multicolumn{3}{l}{$\begin{array}{l}\text { Table 4: The time given by doctors to the } \\
\text { patients during this pandemic in OPD and IPD }\end{array}$} \\
\begin{tabular}{lcc}
\hline \multicolumn{1}{l}{ Time spent with } \\
patients [minutes]
\end{tabular} & OPD & \\
\hline Less than 5 & $49(41.5 \%)$ & $29(24.6 \%)$ \\
$5-10$ & $44(37.3 \%)$ & $46(39 \%)$ \\
$10-15$ & $9(7.6 \%)$ & $32(27.1 \%)$ \\
More than 15 & $13(11 \%)$ & $4(3.4 \%)$ \\
Not applicable & $3(2.5 \%)$ & $7(5.9 \%)$ \\
\hline
\end{tabular}

\begin{tabular}{|c|c|}
\hline Time dedicated to patients & $\mathbf{n}$ \\
\hline Same as pre-pandemic & $12(10.2 \%)$ \\
\hline Increased & $19(16.1 \%)$ \\
\hline Reduced & $61(51.7 \%)$ \\
\hline Depends on case & $25(21.2 \%)$ \\
\hline
\end{tabular}

\begin{tabular}{lc} 
Table 6: The changes noted in procedures or \\
surgeries by doctors during this pandemic, in \\
comparison to pre pandemic \\
\hline Changes in procedures or surgeries & $\mathbf{n}$ \\
\hline Same as before & $20(16.9 \%)$ \\
Reduced number of elective procedures/surgeries & $39(33.1 \%)$ \\
Only emergencies or acute care & $32(27.1 \%)$ \\
Variable & $26(22 \%)$ \\
\hline
\end{tabular}

Health care worker at risk for COVID-19 infection is shown in Table 10.

The impact of COVID-19 pandemic in clinical practice over long term is shown in Table 11.

The concerns of doctors about the COVID-19 pandemic on the clinical practice are shown in Table 12.

The aspect of doctors' life that is affected by the COVID pandemic on the clinical practice is shown in Table 13.

Doctors posted in COVID/triage area on rotation: Table 14.

\begin{tabular}{lc}
$\begin{array}{l}\text { Table 7: Telemedicine advice given by doctors } \\
\text { during the pandemic }\end{array}$ \\
\hline Telephone consultations per day & $\mathbf{n}$ \\
\hline$<5$ & $85(72 \%)$ \\
$5-10$ & $19(16.1 \%)$ \\
$10-20$ & $6(5.1 \%)$ \\
$>20$ & $3(1.5 \%)$ \\
\hline
\end{tabular}

\begin{tabular}{lc}
$\begin{array}{l}\text { Table 8: Table showing the doctors' opinion on } \\
\text { telemedicine }\end{array}$ \\
\hline Telemedicine usefulness & $\mathbf{n}$ \\
\hline Helpful in manageable cases & $49(41.5 \%)$ \\
Difficult to analyze over phone & $42(35.5 \%)$ \\
In addition OPD evaluation must & $10(8.5 \%)$ \\
Rarely opine/donot give opinion & $15(12.7 \%)$ \\
\hline
\end{tabular}

\begin{tabular}{lc} 
Table 9: The type of personal protection \\
measures used by doctors during the pandemic \\
\hline Personal protective measures & $\mathbf{n}$ \\
\hline Only face shield/mask & $26(22 \%)$ \\
Face shield/mask with gloves & $70(59.3 \%)$ \\
Face shield/mask with gloves with surgical gown & $14(11.9 \%)$ \\
Full PPE & $6(5.1 \%)$ \\
\hline
\end{tabular}

\begin{tabular}{lc} 
Table 10: Opinion of doctors about risk of \\
contracting COVID infection at the workplace \\
\hline Place of work and risk & $\mathbf{n}$ \\
\hline Same for all and everywhere in hospital & $37(31.4 \%)$ \\
Triage & $36(30.5 \%)$ \\
COVID/SARI & $32(27.1 \%)$ \\
Surgeries/procedures & $12(10.2 \%)$ \\
\hline
\end{tabular}

Table 11: The impact of COVID-19 pandemic in clinical practice over long term

\begin{tabular}{lc} 
Impact & $\mathbf{n}$ \\
\hline Negative & $46(39 \%)$ \\
Revert to pre-pandemic & $35(29.7 \%)$ \\
Impossible to return topre-pandemic & $21(17.8 \%)$ \\
Positive & $16(13.6 \%)$ \\
\hline
\end{tabular}

Asian Journal of Medical Sciences | Mar 2022 | Vol 13 | Issue 3 


\begin{tabular}{lc} 
Table 12: Table showing the concerns of \\
doctors about the COVID-19 pandemic on their \\
clinical practice \\
\hline Primary concern & $\mathbf{n}$ \\
\hline Financial problems & $11(9.3 \%)$ \\
Problems in handling complicated cases & $15(12.7 \%)$ \\
Patient care is affected & $31(26.3 \%)$ \\
Multifactorial & $60(50.8 \%)$ \\
\hline
\end{tabular}

\begin{tabular}{lc} 
Table 13: Table showing the aspect of doctors' \\
life that is affected by the COVID pandemic \\
\hline Aspect of life affected & $\mathbf{n}$ \\
\hline Family life & $23(19.5 \%)$ \\
Lifestyle/habits & $28(23.7 \%)$ \\
Psychological distress/perceived well-being & $37(31.4 \%)$ \\
Patient doctor relationship and security & $30(25.4 \%)$ \\
\hline
\end{tabular}

\begin{tabular}{lc}
\multicolumn{2}{l}{ Table 14: Table showing the Doctors postings in } \\
COVID/triage area on rotation \\
\hline Postings & $\mathbf{n}$ \\
\hline Based on the surge in cases & $48(40.2 \%)$ \\
Monthly & $34(28 \%)$ \\
Weekly & $15(12.7 \%)$ \\
Never worked & $21(17.8 \%)$ \\
\hline
\end{tabular}

The risk of COVID-19 infection exposure risk for the doctors is shown in Table 15.

The opinion of doctors on patients/attendees following the COVID-19 protocol [masks and social distancing] during pandemic: Table 16.

The COVID pandemic impact on clinical examination technique during the pandemic in practice is shown in Table 17.

The COVID-19 protection protocol followed by doctors during the surgeries/procedures: Table 18.

The follow-up advice given to the patients (general cases, post-operative, and any chronic case) by doctors: Table 19 .

The opinion of doctors on the use of investigations during the pandemic in their clinical practice is shown in Table 20.

The behavior of patients/attendees during pandemic is shown in Table 21.

In our study, 46 doctors (39\%), the participants of the study, were not infected with COVID-19 infection during and till the study period, whereas remaining $61 \%$ were infected one time or the other since the onset of pandemic. Forty-four doctors (37.3\%), the participants,

\begin{tabular}{|c|c|}
\hline Risk & $\mathbf{n}$ \\
\hline Some risk & $13(11 \%)$ \\
\hline Avoidable using PPE & $65(55.1 \%)$ \\
\hline $\begin{array}{l}\text { High if managing the COVID patients even with } \\
\text { PPE }\end{array}$ & $19(16.1 \%)$ \\
\hline High in non-COVID side & $21(17.8 \%)$ \\
\hline
\end{tabular}

\begin{tabular}{l} 
Table 16: Table showing the opinion of doctors \\
on patients/attendees following the COVID-19 \\
protocol [masks, social distancing] during \\
pandemic \\
$\begin{array}{l}\text { Protection measures followed } \\
\text { by patients/attendees }\end{array}$ \\
\hline $\begin{array}{l}\text { Partially } \\
\text { Ignorant }\end{array}$ \\
$\begin{array}{l}\text { Never } \\
\text { Strictly }\end{array}$ \\
\hline
\end{tabular}

\begin{tabular}{|c|c|}
\hline Clinical examination technique & $\mathbf{n}$ \\
\hline & $16(13.6 \%)$ \\
\hline Only essential examination is done & $78(66.1 \%)$ \\
\hline Avoid touching the patients & $14(11.9 \%)$ \\
\hline Only vitals are examined & $6(5.1 \%)$ \\
\hline
\end{tabular}

Table 18: COVID-19 protection protocol followed by doctors during the surgeries/procedures

\begin{tabular}{lc}
\hline Opinion & $\mathbf{n}$ \\
\hline Follow in all cases & $64(54.2 \%)$ \\
Selected cases & $21(17.8 \%)$ \\
While managing in COVID cases & $13(11 \%)$ \\
Not using & $8(6.8 \%)$ \\
Not applicable & $11(9.3 \%)$ \\
\hline
\end{tabular}

\begin{tabular}{lc}
$\begin{array}{l}\text { Table 19: Table showing the follow up protocols } \\
\text { followed by the doctors }\end{array}$ \\
\hline Follow-up advice changes in clinical practice & $\mathbf{n}$ \\
\hline No change in comparison to pre-pandemic & $22(18.6 \%)$ \\
Reduced number of follow-up visits & $63(53.4 \%)$ \\
Teleconsultation & $23(19.5 \%)$ \\
Follow-up with a doctor near home/local & $7(5.9 \%)$ \\
\hline
\end{tabular}

opined that the infection was contracted during the clinical practice. Nine $(7.6 \%)$ doctors believe probably the source of infection was outside the clinical practice/ hospital (social gatherings, market, etc.). Nineteen $(16.1 \%)$ doctors though infected are not sure about the probable place/source of contracting COVID-19 infection. 


\begin{tabular}{l} 
Table 20: Table showing the opinion of \\
doctors on the use of investigations during the \\
pandemic in their clinical practice \\
\hline $\begin{array}{l}\text { Use of investigations in comparison to the pre } \\
\text { pandemic, in clinical practice }\end{array}$ \\
\hline $\begin{array}{l}\text { Unchanged } \\
\text { Use and reliability on investigations has increased }\end{array}$ \\
$\begin{array}{l}\text { Use and reliability on investigations has decreased } \\
\text { Only reliability has increased }\end{array}$ \\
\hline
\end{tabular}

\begin{tabular}{lc}
$\begin{array}{l}\text { Table 21: The behavior of patients/attendees } \\
\text { during pandemic }\end{array}$ \\
\hline Behavior of patients/attendees & $\mathbf{n}$ \\
\hline Unaltered & $30(25.4 \%)$ \\
Polite & $21(17.8 \%)$ \\
Aggressive & $44(37.3 \%)$ \\
Casual & $22(18.6 \%)$ \\
\hline
\end{tabular}

\section{DISCUSSION}

Our study shows a significant reduction in nonCOVID cases seen by doctors in the OPD during the pandemic in comparison to the pre pandemic. A study by Homeniuk and Collins noted more than half of practices saw a reduction in the non-COVID-related consultations. ${ }^{1}$ A German study with 1095 general practices (GPs) and patients aged 65 or older found that there was a $14 \%$ decrease in consultations in May 2020 compared with the same time in $2019 .{ }^{1}$ Another article says that there was a decrease overall in GP appointments (GPs and practice nurses) in the early days of the pandemic. This is similar to reports in the UK from data taken from March 2020, where the National Health Service has noticed a $30 \%$ decrease in the number of GP appointments compared with previous data. ${ }^{5}$ An online survey by Keshav et al., with respect to Indian orthopedic practice during the COVID-19 pandemic, noted that theroutine OPDs were shut down in most of the states, more than half of practicing surgeons noted fall in OPD numbers by $90 \%$, reasons for fall in OPD patients numbers - doctors did not take risk in seeing cases without screening for COVID, patients' fear of contracting infection, inability to reach the hospital due to cancellation of public transportation, and older people with chronic comorbidities willing to take over the counter medications staying at home. ${ }^{3}$

Our study noted a reduction in the number of nonCOVID IPD cases during the COVID pandemic in comparison to the pre-pandemic IPD cases. Beshyah et al., study has reported a significant reduction in inpatient admissions during the pandemic period compared to pre-pandemic time resulting in a flat rate for empty beds and reduced hospital revenue. ${ }^{6}$ A study by Birkmeyer et al., noted hospital admissions fell precipitously with the onset of COVID-19 pandemic in the US in March 2020, with hospital admissions < $50 \%$ of the capacity. It was hypothesized that many patients did not seek medical care out of fear of contagion, stay at home orders, or concerns about access at COVID-19 overrun hospitals. ${ }^{7}$

In our study, doctors from various specialties were managing COVID/SARI-related cases during the pandemic. The other cases that were seen by doctors during this pandemic were emergencies concerned to their specialty, cases referred from other hospitals for further management, and increase in cross consultations. It was noted in the Nair AGstudy that most of the ophthalmologists working in large institutes and government hospitals were still seeing and operating cases. Majority of the private practitioners had closed their practices and were not actively involved in direct patient care. ${ }^{2}$ While emergencies such as trauma and other conditions that qualified for emergent care remained the most common procedures still being performed. ${ }^{2}$

In our survey, $43.2 \%$ of doctors were managing SARI/ COVID-related cases during the pandemic, $19.5 \%$ say that they were engaged in managing acute cases of their specialty. A survey by Keshavet al., noted, emergency cases as a result of orthopedic trauma have reduced in this pandemic due to reduced road traffic accidents. One thing which is followed in pandemic is to get RTPCR COVID testing for patients and take for surgery or managing the cases conservatively till the report is negative. ${ }^{3}$

In our study, all the doctors utilized telemedicine during the pandemic. The majority of these limited the teleconsultations to $<5$ per day. The doctors opinion, $41 \%$, in our survey opine telemedicine is helpful in manageable cases, however, one-third opine it is difficult to analyze over phone. A study by Homeniuk and Collins states that the rapid national adoption of telemedicine consultations as well as electronic prescribing in Ireland has presented an opportunity; however, GPs are concerned that without as much face-to-face contact, critical non-verbal communication is missing from consultations, and insome cases, telephone consults are insufficient to address patient concerns. ${ }^{1}$ The telemedicine may have negative impact on delivering the health care to those people who have poor computer proficiency, the elderly,and the people from lower socioeconomic strata. ${ }^{1}$ 
In our study, majority of the doctors used face mask and gloves in their clinical practice. A study by Keshav et al., says that all the surgeries should be done wearing PPE kits. ${ }^{3}$ A study by Nasta et al., noted that $56.9 \%$ of doctors would use PPE in all cases. ${ }^{4}$

In our study, $56.8 \%$ of doctors believe that the pandemic will have negative impact on the practice and it may not be possible to revert to pre-pandemic practice. A study by Homeniuk and Collins noted the changes adopted during this pandemic accelerated the digitalization of healthcare and it is likely that the way GP is delivered will not return to as it was before the COVID-19 pandemic and increased telemedicine can be expected. ${ }^{1}$ An article by Rubin mentions after the ease of lockdown physicians practice is somewhat improving but it is still well below pre-COVID-19 pandemic levels and is expected to stay that way, at least as long as social distancing is required. The observed declines in vaccination coverage, during the pandemic,might leave young children and communities vulnerable to diseases such as measles which are vaccine preventable. $^{8}$

In our study, majority of the doctors' concern in clinical practice due to pandemic effect is multifactorial: Finances, patient care, etc. During the pandemic, the economic growth of country is negative. These changes have affected the surgeons' income. ${ }^{3} \mathrm{~A}$ study by Nasta et al., states a drop of more than $75 \%$ of their monthly income experienced by $52 \%$ of surgeons, while $22 \%$ faced $50-75 \%$ reduction. ${ }^{4}$ Health authorities and scientists have warned that widespread misinformation about COVID-19 is a serious concern causing xenophobia worldwide. $^{4}$

Our study has noted that the pandemic impact on practice has affected doctors' family life, lifestyle, wellbeing, and the patient-doctor relationship. A survey by Keshavet al., noted most of the surgeons stopping elective surgeries, leading to patients getting profoundly affected. ${ }^{3}$ de Sutter et al., study noted the challenges in times of COVID-19 pandemic: Shortage of personnel and equipment, stress, loneliness, insecurity, distress, fear for one loved ones, and personal health issues during treating patients. ${ }^{9}$ Psychological distress and well-being in doctors have been the subject of numerous studies done during the COVID-19 pandemic. ${ }^{10}$ An article by Rubin says that the pandemic has affected the smaller clinical practices to close, significant income reduction in clinical practice and hence reduced salary to the nurses working in these clinics. ${ }^{8}$
In our survey, the risk of $\mathrm{HCW}$ getting infected with COVID-19 infection is same for all and risk is everywhere in the clinical practice. However, $57.6 \%$ of doctors in our study believe that it is more for $\mathrm{HCW}$ working in triage or SARI or COVID area.

In our study, $40.7 \%$ of doctors work in COVID or triage area on a weekly or monthly rotation basis during the pandemic. In our study, two-thirds of the doctors belonged to junior cadre. An article on COVID-19 impact on medical trainees states that the number of patients attending the clinics was reduced, and therefore, the opportunities for trainee engagement in the outpatient environment were significantly less. The medical trainees were reassigned from team-based specialty rotations to non-specialty specific general medicine wards. ${ }^{11}$

In our survey, majority of the doctors opined that the COVID-19 infection in clinical practice is avoidable using PPE. Our survey has noted down that $54.2 \%$ of doctors follow COVID-19 protection protocol for all cases during the procedures/surgeries, $28.8 \%$ say that they follow only in selected cases. A study by Keshav et al., mentions considering the fact that RT-PCR has a false-negative rate of almost $20 \%$, all the surgeries should be done wearing PPE kits. ${ }^{3}$

In our study, one-third doctors opined that the number of elective surgeries are reduced during the pandemic. The next common change observed during the pandemic is managing only acute cases. The pandemic has made an impact on follow-up of patients too, $79 \%$ of doctors' advice the patients to follow-up less frequently or advise telemedicine or the patients are advised to follow-up in a local hospital. A survey on orthopedic practice in pandemic reported significant cancellation of orthopedic surgical activities including emergency and trauma surgeries. Other reports from urology specialties also documented progressively reduced interventions, changes in patients' and surgical scheduling, cancellation of elective surgeries, and staff recruitment from different medical and surgical specialties in managing and treating COVID-19-infected patients. ${ }^{6}$

The pandemic has made an impact on clinical examination pattern in clinical practice. In our survey, two-third of the doctors opined only the essential examination is carried out. The use and reliability on investigations in this pandemic have increased as per $66.8 \%$ of the doctors in our study. In our survey, $51.7 \%$ of doctors have opined that the time dedicated to the patients in OPD and IPD during the pandemic in comparison to pre-pandemic has 
reduced. Beshyah et al., article states before the pandemic, an average of 30 patients in a $24 \mathrm{~h}$ period was referred for evaluation. The majority (93.3\%) of these resulted in hospital admission. During the COVID-19 pandemic, an average of 52 patients was assessed per $24 \mathrm{~h}$ period, mostly with respiratory symptoms; fewer assessments resulted in admission $(53.8 \%)^{6}$

In our survey, $57.7 \%$ of doctors opined that patient and their attendees partially followed or never follow the COVID-19 protection protocol. The behavior of the patients and their attendees during the pandemic has been aggressive as $37.3 \%$ of doctors opined. Bhat et al., article on dental practice during the pandemic, mentions the guidelines to be followed by patients: Hand hygiene, cough etiquette, social distancing in waiting area, face mask, and designated waste disposal area in hospital. ${ }^{12}$

In our study, majority of the doctors opined that they contracted infection during the clinical practice. An eye clinic is a high-risk environment for both: The examining doctor and the patient. ${ }^{2}$ The majority of orthopedic procedures are aerosol generating, could be a potential source of infection to the surgeons in OT. ${ }^{3}$

\section{Limitations of the study}

The limitations of our study are it includes the clinicians working in medical and dental college, doctors in private practice are not included. The study was done during the later phase of the second wave of COVID-19. The study cannot comment on the clinical practice trends after the second wave.

\section{CONCLUSION}

The COVID-19 pandemic impact on clinical practice is varied. The number of non-COVID patients both in OPD and IPD in all specialties has reduced. The doctors from various specialties are involved in managing cases out of their specialty and have noticed reduced number of elective surgeries, however, acute care of the cases remains same. The observed changes in clinical examination pattern are as follows: Reduced time spent with patients, cutting down the detailed examination pattern, and increase in use and reliability on investigations. The pandemic has affected doctors in their family and personal life, patient-doctor relationship, and has affected perceived well-being. The use of telemedicine can be helpful. The points learned from this survey are health care workers should continue to use personal protection measures as per the guidelines andsame should be emphasized persistently to the patients and their attendees. The time is bad during the pandemic, it can be overcome by following the pre-pandemic clinical practice principles.

\section{ACKNOWLEDGMENT}

We would like to thank head of the department, professors, faculty, and residents of general medicine department.

\section{REFERENCES}

1. Homeniuk R and Collins C. How COVID-19 has affected general practice consultations and income: General practitioner cross sectional population survey evidence from Ireland. BMJ Open. 2021;11(4):e044685.

https://doi.org/10.1136/bmjopen-2020-044685

2. Nair AG, Gandhi RA and Natarajan S. Effect of COVID-19 related lockdown on ophthalmic practice and patient care in India: Results of a survey. Indian J Ophthalmol. 2020;68(5): 725-730.

https://doi.org/10.4103/ijo.IJO_797_20

3. Keshav K, Kumar A, Sharma P, Baghel A, Mishra P and Huda N. How much has COVID-19 pandemic affected Indian orthopaedic practice? Results of an online survey. Indian J Orthop. 2020;54(2):358-367.

https://doi.org/10.1007/s43465-020-00218-z

4. Nasta AM, Goel R, Kanagavel M and Easwaramoorthy S. Impact of COVID-19 on general surgical practice in India. Indian J Surg. 2020;82(3):259-263

https://doi.org/10.1007/s12262-020-02443-0

5. The Health Foundation. How Might COVID-19 Have Affected People's Ability to See Their GP? COVID-19 Chart Series. London: The Health Foundation; 2020. Available from: https://www.health.org.uk/news-and-comment/charts-andinfographics/how-might-covid-19-have-affected-peoples-abilityto-see-gp

6. Beshyah SA, Ibrahim WH, Hajjaji IM, Ben Mami F, Arekat M and Abdelmannan DK. Impact of the COVID-19 pandemic on clinical practice, medical education, and research: An international survey. Tunis Med. 2020;98(8-9):610-618.

7. Birkmeyer JD, Barnato A, Birkmeyer N, Bessler $\mathrm{R}$ and Skinner J. The impact of the cOVID-19 pandemic on hospital admissions in the United States: Study examines trends in US hospital admissions during the COVID-19 pandemic. Health Aff (Millwood). 2020;39(11):2010-2017.

https://doi.org/10.1377/hlthaff.2020.00980

8. Rubin R. COVID-19's crushing effects on medical practices, some of which might not survive. JAMA. 2020;324(4):321-323. https://doi.org/10.1001/jama.2020.11254

9. de Sutter A, Llor C, Maier M, Mallen C, Tatsioni A, van Weert H, et al. Family medicine in times of 'COVID-19': A generalists' voice. Eur J Gen Pract. 2020;26(1):58-60. https://doi.org/10.1080/13814788.2020.1757312

10. de Sio S, Buomprisco G, La Torre G, Lapteva E, Perri R, Greco E, et al. The impact of COVID-19 on doctors' well-being: Results of a web survey during the lockdown in Italy. Eur Rev Med Pharmacol Sci. 2020;24(14):7869-7879.

https://doi.org/10.26355/eurrev_202007_22292 
11. Gaffney B, O'Carroll O, Conroy F, Butler MW, Keane MP and McCarthy C. The impact of COVID-19 on clinical education of internal medicine trainees. Ir J Med Sci. 2021;190(2):845-847.

https://doi.org/10.1007/s11845-020-02350-z
12. Bhat PK, Nayana MM and Jayachandra MY. Containing the contagion-dental practice management post-COVID-19. Indian J Dent Sci. 2021;13(1):54-59.

https://doi.org/10.4103/IJDS.IJDS 6320

\section{Authors Contribution:}

Dr Naveen S Kulkarni contributed by formulating the concept and design of the study, acquisition of data, analysis and interpretation of the results. He was also actively involved in drafting the article, revising it critically and final approval of the manuscript. Dr Neelakanth S Patil contributed in collection of data, analysis and interpretation of results and drafting the article. Dr Vishalakshi was involved in the planning of study design, questionnaire, acquisition of data, critically reviewing the article and in final approval. Dr Asha Bullappa, Dr Subhashree Mishra and Dr Hithashree Shriyan were actively involved in data acquisition, analysis of results, statistical analysis, interpretation of results, critical review of the article from time to time.

\section{Work attributed to:}

Department of General Medicine, Shri Dharmasthala Manjunatheshwara College of Medical Sciences and Hospital, Shri Dharmasthala Manjunatheshwara University, Dharwad - 580 009, Karnataka, India.

\section{Orcid ID:}

Dr. Naveen S Kulkarni - (1) https://orcid.org/0000-0002-1219-299X

Dr. Neelakanth S Patil - (10 https://orcid.org/0000-0002-9024-6367

Dr. Vishalakshi - (i) https://orcid.org/0000-0002-0919-9832

Dr. Asha Bullappa - (D https://orcid.org/0000-0002-1567-5241

Dr. Hithashree Shriyan - (D) https://orcid.org/0000-0003-4653-9311

Dr. Subhashree Mishra - (1) https://orcid.org/0000-0003-3336-8218

Source of Support: None, Conflicts of Interest: None. 